University of South Florida

DIGITAL COMMONS

Digital Commons @ University of

@ UNIVERSITY OF SOUTH FLORIDA

South Florida

Rehabilitation and Mental Health Counseling

Faculty Publications

Rehabilitation and Mental Health Counseling

$1-1-2011$

\title{
Wireless telesurveillance system for detecting dementia
}

\author{
William D. Kearns \\ University of South Florida, kearns@usf.edu \\ James L. Fozard \\ University of South Florida, jfozard@usf.edu \\ Vilis O. Nams \\ Nova Scotia Agricultural College \\ Jeffrey D. Craighead \\ James A. Haley Veterans Hospital Center of Excellence
}

Follow this and additional works at: https://digitalcommons.usf.edu/mhs_facpub

Part of the Psychology Commons

\section{Scholar Commons Citation}

Kearns, William D.; Fozard, James L.; Nams, Vilis O.; and Craighead, Jeffrey D., "Wireless telesurveillance system for detecting dementia" (2011). Rehabilitation and Mental Health Counseling Faculty Publications. 3.

https://digitalcommons.usf.edu/mhs_facpub/3

This Article is brought to you for free and open access by the Rehabilitation and Mental Health Counseling at Digital Commons @ University of South Florida. It has been accepted for inclusion in Rehabilitation and Mental Health Counseling Faculty Publications by an authorized administrator of Digital Commons @ University of South Florida. For more information, please contact digitalcommons@usf.edu. 


\title{
Wireless telesurveillance system for detecting dementia
}

\author{
William D. Kearns PhD \\ Department of Aging and Mental Health Disparities, \\ Louis de la Parte Florida Mental Health Institute, College of \\ Behavioral and Community Sciences, University of South Florida, \\ Tampa, Florida 33612, USA \\ E: kearns@usf.edu \\ James L. Fozard PhD \\ School of Aging Studies, College of Behavioral and Community \\ Sciences, University of South Florida, Tampa, Florida 33612, USA \\ Vilis O. Nams PhD \\ Department of Environmental Sciences, Nova Scotia Agricultural \\ College, Truro, Novo Scotia, Canada \\ Jeffrey D. Craighead PhD \\ James A. Haley Veteran's Hospital, HSR\&D/RR\&D \\ Center of Excellence, Tampa, Florida, USA
}

W.D. Kearns, J.L. Fozard, V.O. Nams, J.D. Craighead. Wireless telesurveillance system for detecting dementia. Gerontechnology 2011; 10(2):90-102; doi:10.4017/ gt.2011.10.2.004.00 Objective We hypothesized path tortuosity (an index of casual locomotor variability) measured by a movement telesurveillance system would be suitable for assisted living facility residents clinically diagnosed with dementia. Background We examined the relationship of dementia to path tortuosity and to movement speed and path length variability, both of which increase in dementia. Methods Daytime movements of 25 elders (19 female; 14 with dementia; average age 80.6) were monitored for 30 days using radio transponders measuring location with a maximum accuracy of $20 \mathrm{~cm}$. After 30 days, the Mini Mental State Exam (MMSE) and Revised Algase Wandering Scale-Community Version (RAWSCV) were administered. Results Fractal Dimension (Fractal D), a measure of path tortuosity, correctly classified all but 2 residents with dementia; sensitivity 0.857 , specificity 0.818 while the MMSE had 6 misclassifications, a sensitivity of 0.857 and a specificity of 0.727 . Individual logistic regressions of dementia diagnosis on predictors MMSE and Fractal D were significant, but a logistic regression using both predictors found Fractal D marginally predictive of dementia $(p=0.055)$ while the MMSE was not $(p=0.168)$. Although significantly correlated with Fractal $D$, rate of travel and mean path distance were not predictive of dementia. Fractal D correlated negatively with overall MMSE $(r=-0.44, n=25, p<0.05)$ but the relationship was mediated by MMSE Geographical Orientation items. Fractal D was unrelated to the RAWS-CV. Conclusions Telesurveillance-measured path tortuosity is greater in persons diagnosed with dementia. Persons with dementia have relatively more impaired spatial memory which is required for successful navigation. Application Automatic monitoring of direction, length and speed of unconstrained movements.

Keywords: dementia, wandering, tracking technology, path tortuosity

A variety of procedures have been used to identify irregularities in gait in elderly persons with cognitive declines and dementia. The present paper describes an automatic indoor movement tracking system that measures changes in path direction as well as rate and duration of movement. Gait irregularities have been observed to distin- 


\section{Detecting dementia}

guish 'normal' or benign memory problems associated with normal aging from those linked to a clinical diagnosis of dementia. Changes in gait and movement patterns have been observed before and after clinical diagnoses of dementia ${ }^{1}$. In a study of five years of clinical records of symptoms identified in patients with preclinical dementia, gait disturbances were the earliest predictor followed by cognitive complaints ${ }^{2}$. A recent longitudinal study of preclinical cognitive decline occurring prior to dementia diagnosis identified three cognitive functions that changed prior to diagnosis ${ }^{3}$. The order of preclinical declines were, 3, 2, and 1 year prior for Visuospatial, Global, and Memory, respectively. Visuospatial changes were assessed with psychological test performance in this study but they also reflected floor maze navigation variations in other studies ${ }^{4}$.

\section{MOVEMENT VARIABILITY}

For persons with a clinical diagnosis of dementia, two lines of published research link increased movement variability to dementia. In the first, multiple trained human observers classified routine nursing home resident ambulation as either purposeful traveling, for instance, going to the dining room, or as falling into one of three categories of aimless locomotion (wandering): random, lapping and pacing ${ }^{5 p .77}$. Over three observation periods, residents with dementia had more random elements (wandering) in their paths. This study was the first in a series culminating in the creation of the 40 item Revised Algase Wandering Scale-Community Version (RAWS-CV) ${ }^{6}$. The items in the RAWS$\mathrm{CV}$ were organized into subscales, two of which were called persistent walking and repetitive walking. In addition there is a single question, "Is this person a wanderer?" The categories developed in the earlier research -random, lapping and pacing- mostly migrated to these two subscales. The individual items in the two subscales concerned both the aimless aspect of walking, for instance, the person walks aimlessly and has difficulty way-finding, and the amount and quality of the wandering, for instance, restlessness and walking between meals and at night.
In the second line of research evidence, variability in stride to stride gait speed and length is measured as elders walk on prescribed paths. Increased variability in these measures correlates negatively with cognitive performance measures, including the MMSE. Hayes and colleagues ${ }^{7,8}$ measured daily variations in walking speed in free voluntary movements of 14 seniors living in their own homes. They deployed wall mounted PIR (Passive InfraRed) sensors which were triggered as a person walked past the monitored wall, allowing the estimation of travel speed. Variability in walking speeds and activity levels were greater in the 7 participants with mild cognitive impairment than in the 7 without the diagnosis, results which extend earlier findings using gait mats ${ }^{9-12}$ to voluntary movements observed in a home setting.

\section{Path tortuosity}

Path tortuosity (number of changes in direction of successive movement paths) has been measured during routine ambulation of elderly residents in the common indoor living spaces of congregate living facilities called Assisted Living Facilities (ALF). An ALF is a congregate living facility, usually for elderly persons, that provides hotel services and assistance with activities of daily living (ADL) but no in-house medical or nursing support. Path tortuosity was measured with a highly accurate geographical localization device described in research by Kearns et al. ${ }^{13,14}$ and Kearns and Fozard ${ }^{15}$. An active radio frequency identification (RFID) transponder reports position with $20 \mathrm{~cm}$ accuracy in $\mathrm{x}, \mathrm{y}$, and $z$ dimensions when the wearer passes within $150 \mathrm{~m}$ of fixed sensors, and is capable of position updates at rates exceeding 10/s. Transponder position, the path's points of origin and completion, the travel duration, speed and vector are obtained using this method. A summary measure, Fractal Dimension (Fractal D), used in movement ecology studies to characterize exploratory behavior in numerous species ${ }^{16}$, is employed to characterize path tortuosity. Fractal D ranges from 1 where a path follows a perfectly straight line (requiring only a single dimension, length, to describe it) to a value 


\section{Detecting d e me ntia}

of 2 where the path is so tortuous (chaotic) that it completely covers the plane of movement and requires all of a second dimension (width) to describe it. In contrast to the fractal indices employed by Hausdorff ${ }^{10}$ to describe stride time variability on prescribed courses and standardized gait and balance assessments (SGB), which may define spatial variability in terms of variations in length of successive strides or number of times that the location of a step falls outside a specified path $^{17}$, we operationally define spatial variability in terms of the tortuosity of paths measured by Fractal D. Fractal D provides spatial information characterizing locomotion occurring over wide areas and from persons unable to complete normal SGB protocols due to mobility constraints (i.e. wheelchair confinement or the use of walkers). Fractal $D$ recorded from the aimless locomotion of frail elders in assistive living facilities correlates negatively and significantly with MMSE scores $(r=-0.47 ; n=14 p=0.05)^{15}$. Unlike the work of Hayes, Pavel and colleagues, the present system, based on RFID technology requires that participants wear small transponders that emit signals to a sensor array; coverage is limited to the monitored spaces.

\section{ReseARCH GOALS}

The research goals were to: (i) determine if path tortuosity was greater for ALF residents with a clinical diagnosis of dementia than for residents without the diagnosis; (ii) describe the relationship between component measures of path length and rate of travel to dementia; (iii) explore the relationship of a measure of wandering behavior gathered by human observers, the Revised Algase Wandering Scale-Community Version (RAWS$\mathrm{CV})^{18,19}$, to path tortuosity; (iv) explore the relationship of path tortuosity to a widely used measure of cognitive functioning, the Mini Mental State Exam (MMSE), and determine the unique variance Fractal D and the MMSE contribute to differentiating dementia diagnoses in ALF residents, and (v) obtain a fuller understanding of the documented negative association of MMSE to Fractal D by determining the MMSE geographic and temporal orientation items' correlation with Fractal D.
Based on a review by Luis and Brown ${ }^{20}$ we hypothesized the MMSE 'Geographic Orientation' component would be strongly positively correlated with Fractal D scores. The geographical orientation items in the MMSE require the participant to name the state, county, city, name of the ALF facility, and type of facility they reside in.

By definition the movement patterns generated in common locomotion are composed of a number of paths with varying path directions and distances. The rate of travel is inversely related to the number of changes in path direction and positively related to the total distance traveled. We examined the correlations between movement speed and path length to dementia.

\section{Movement eCOlOGy PARADIGM}

The movement ecology paradigm provides a theoretical framework to analyze human path tortuosity; it is a transactional analysis linking an individual's internal state, their navigational capacity and their motion capacity with features in their external environment ${ }^{21 p 10954}$. Each change in location, termed a 'movement path', changes the person-environment dynamic potentially altering any or all capacities of the individual. In the present study, the internal state, or 'why move', is defined by the goal of traversing a common living space for a meal, getting to a sleeping area, or engaging in recreation. Navigational capacity, having the ability to determine 'where to move', is differentially affected by the presence of dementia and differences in cognitive abilities. Motion capacity, knowing 'how to move', applies to independently mobile persons or those who move with the aid of a walker, or a wheelchair.

Dementia may affect navigational capacity either by changing orientation or attention. Luis and Brown ${ }^{20}$ identified studies supporting the hypothesis that disordered spatial orientation was responsible for dementia related wandering ${ }^{22,23}$, and others indicating that difficulty shifting attention -an executive function- was responsible for wandering ${ }^{24,25}$. Both hypotheses are consistent with observa- 


\section{Detecting d e mentia}

tions of higher movement path variability in elderly with clinical diagnoses of dementia.

Another way dementia can affect path tortuosity is through its effect on motion capacity or 'how to move'. Stride to stride gait speed and length, measured when elders walk prescribed paths, correlates negatively with cognitive measures, such as the MMSE, in normal aged and in subjects with dementia $^{9,12}$. Standardized gait and balance assessments (SGB) include stride length, step length, support base, step time, swing time, stance time, single support time, double support time and average velocity measures ${ }^{26,27}$. Static balance assessment includes body sway measures recorded standing on one or two legs with eyes open or closed; dynamic balance assessments are taken walking and performing an additional task such as talking on a cell phone. Recently researchers ${ }^{9,11}$ have employed fractal analytic techniques to SGB thereby unveiling gait and balance variability information leading to improved fall prediction. Hausdorff et al. ${ }^{9}$ have found that increased stride time variability predicted heightened fall risk in community dwelling elders; stride time variability in this study also correlated negatively $(-0.47)$ with participants' MMSE scores.

All three movement ecology paradigm hypotheses (why move, where to move and how to move) predict dementia will increase movement path tortuosity through its degenerative neurological effects on structures controlling motivation, navigational abilities, and skeletal muscle activity.

\section{Method}

\section{Participants}

Participants were 25 volunteers (19 female) recruited from two local ALF sites with ages ranging from 59 to 93 years (mean 80.6, $\mathrm{SD}=9.5)$, capable of independent movement with or without assistive devices. Those with serious mental health disorders (i.e. Schizophrenia, Bipolar Disorder or Developmental Disability) were excluded. ALF records identified 14 with dementia diagnoses (Table 1); severity or duration of dementia was not specified; 7 were fully ambulatory, 11 used wheelchairs, and 7 used a rolling walker.

Records identified those needing assistance with ambulation, bathing, dressing, toileting, eating, grooming and transferring. The type and level of assistance needed was not recorded. Wheelchair use, and ALF staff judgment of whether the participant was a wanderer according to the RAWS-CV was taken into account (Table 1).

\section{Equipment}

Movement data was collected by a Ubisense, Inc. Ultra Wideband RFID research pack with 25 'compact tag' transponders measuring $38 \mathrm{~mm} \times 39 \mathrm{~mm} \times 16.5 \mathrm{~mm}$, weighing $25 \mathrm{~g}$ and secured to the wrist with a comfortable wristband ${ }^{28}$. A Belkin, Inc. Power of Ethernet 100 BaseT switch, 7 shielded category 5e network cables and four wall mounted sensors were placed at each ALF. The sensor network transferred data to a Dell Inspiron model 1501 notebook computer running Ubisense 2.0 realtime location analysis software for processing and storage.

\section{Monitored areas and sensor installation}

One Ubisense sensor was installed at each corner of approximately rectangular common use spaces at each ALF; $25.6 \mathrm{~m}$ by $9.3 \mathrm{~m}$ - site 1 ; and $23.8 \mathrm{~m}$ by $9.4 \mathrm{~m}$ - site 2 (Figure 1). The spaces contained sofas, chairs, tables, and a television and served as gathering places for recreational and social activities, a conduit between dormitory wings and dining rooms and as a passageway to exterior doors which led to covered outdoor porches with chairs and tables. In both sites, furniture was often rearranged to accommodate different events, such as musical activities.

\section{Procedure}

Following University of South Florida Internal Review Board approval, prospective participants and caregivers (for proxy consent) were recruited by one investigator (JLF) who obtained informed consent and proxy consent and informed them the study's intent was to examine elderly persons' movement patterns, and their task would be to wear a 


\section{Detecting dementia}

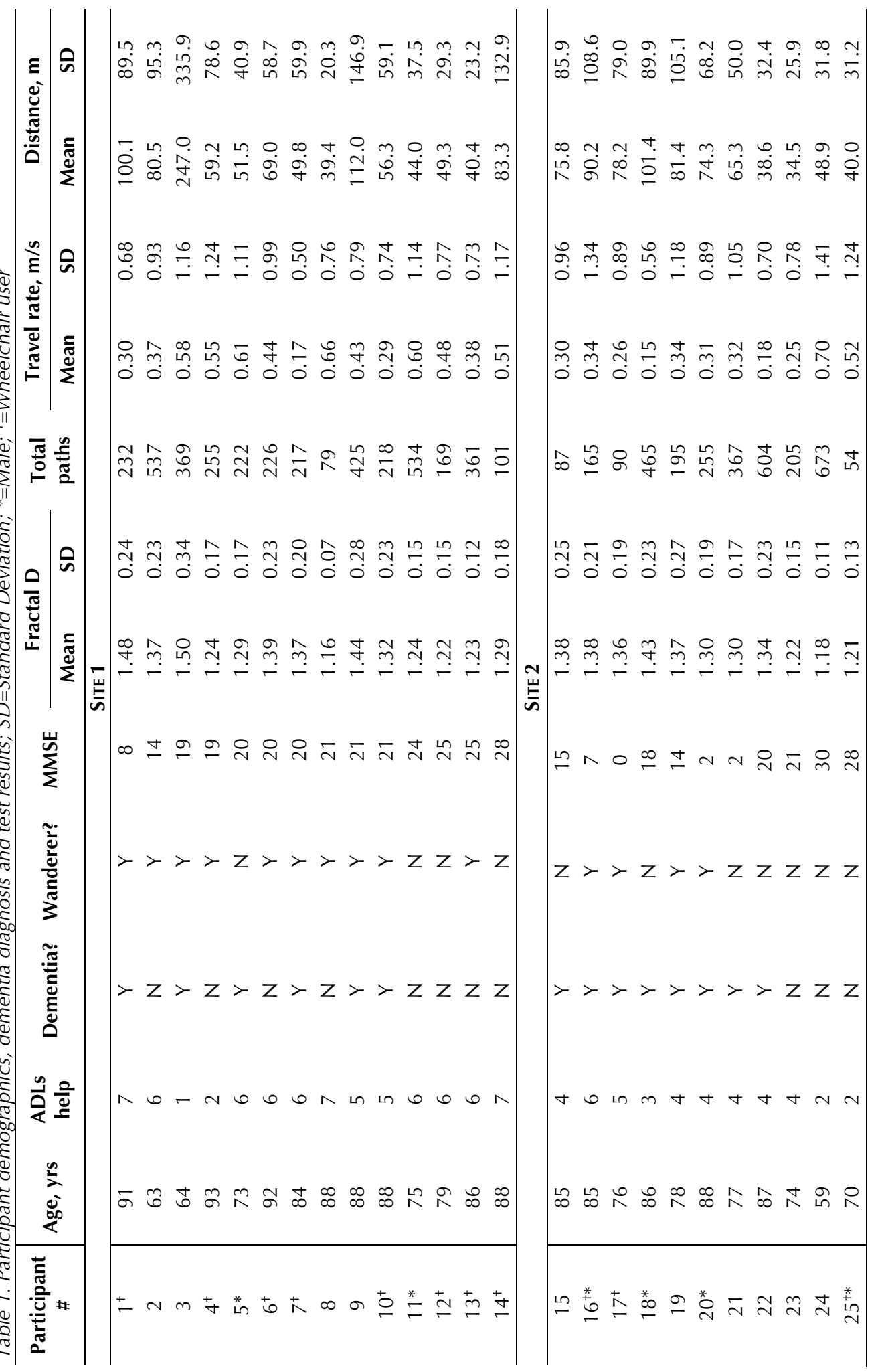




\section{Detecting dementia}
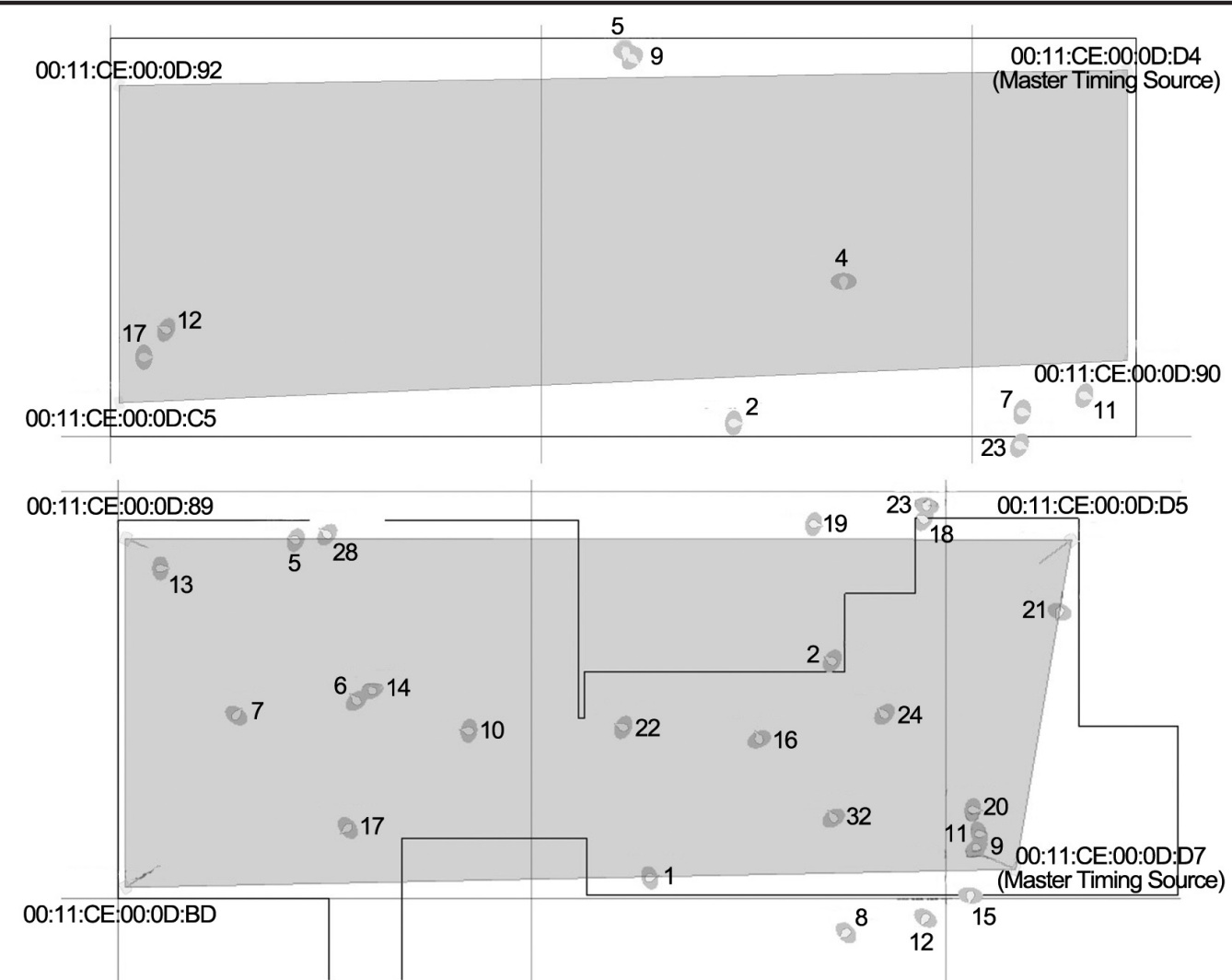

Figure 1. Floor plans for research site 1 (top) and 2 (bottom); sensor locations are at the vertices of the shaded regions and the origin is in the lower left; major divisions are $10 \mathrm{~m}$ increments; individual participants appear as numbered ovals

small comfortable transponder during waking hours for 30 days of recording. Participants were informed the device was safe, would emit very weak radio waves (about $1 / 10^{\text {th }}$ the power of a common cellular telephone) and that they could discontinue the study at any time they desired, but that they should wear it during waking hours but remove it for bathing. By design, the remaining three investigators were blind to identity, age, gender, diagnosis of dementia and mobility aids used by participants.

\section{Experimental protocol}

Transponders were fastened by a comfortable wristband by ALF staff each morning. When in motion, the tags transmitted at 0.43 second intervals $x, y$, and $z$ coordinates in centimeters relative to a fixed origin in one corner of the room. After 30 days of data collection, a trained gerontology graduate student blind to the protocol administered the MMSE. A measure of participant wan- dering behavior, the Revised Algase Wandering Scale-Community Version (RAWS$\mathrm{CV})^{19}$ was independently gathered by an ALF staff member familiar with the participant's normal behavior but blind to MMSE and Fractal D measures.

\section{Data reduction and analyses}

Fractal dimension

Approximately 1.4 million and 1.2 million observations, respectively, were generated at sites 1 and 2. Earlier results ${ }^{14}$ showed transponder radio reflections occurring close to corners and walls affected accuracy compared to those in open areas, therefore clearly impossible data (i.e. indicating a transponder had passed through a solid wall) were eliminated. Similarly, observations outside the monitored area regardless of accuracy were rejected, leaving 855,377 observations from site 1 and 815,960 from site 2. Each observation included transponder number, the date and time (accurate to 0.001s), and the $x, y$, and 


\section{Detecting dementia}

z coordinates relative to each room's fixed origin. To convert the observations to paths, the rule was: (i) a transponder not changing position for 60s or more was stationary; (ii) the first datum in response to movement began a new path; (iii) successive observations accrued until the transponder was stationary for 60s, signifying the path's end.

Path measures included its duration, length and tortuosity measured by the RTFPA described in the next section. Travel rate was determined by dividing path length by its elapsed time and averaging to obtain a mean and standard deviation for each participant. Mean path distance was computed by summing path lengths generated for each participant over 30 days and dividing by the number of paths.

\section{Fractal Dimension}

For each participant Fractal D was calculated dynamically for each path and averaged to produce a single participant score using the Real-Time Fractal Path Analysis (RTFPA) algorithm based on the Adjusted D4 algorithm presented by Nams ${ }^{29,30}$. RTFPA ${ }^{31}$ uses a dynamic programming approach to reduce the memory complexity of the fractal calculation. The data necessary to compute Fractal $\mathrm{D}$ includes the last point received, the total number of points in a path, the measured path length, and the scaling multipliers. RTFPA uses two divider sizes and estimates them dynamically by multiplying the average step length by scale multipliers. Scaling multipliers of 0.5-10 were chosen for the Fractal D algorithm, based on prior observations of elder locomotion using this technology ${ }^{15}$.

\section{Revised Algase Wandering Scale-CV}

The RAWS-CV is a 40-item scale with five subscales measuring persistent walking, repetitive walking, elopement behavior, spatial disorientation and negative outcomes. The RAWS-CV's validation item 'The person is a wanderer', is scored 'no', 'yes, but it is not a problem', 'yes, and it is a problem', 'yes, and it is a major problem'; and was dichotomized to a simple yes/no for this study. Pearson product moment correlations of average rate of travel, RAWS-CV dichotomized validation item and subscales were calculated to evaluate their relationship to Fractal D path variability.

\section{MMSE}

The MMSE full scale and Geographic Orientation and Temporal Orientation subscale scores for each participant were obtained as well as Pearson product moment correlation coefficients calculated with Fractal D, travel rate and RAWS-CV measures.

\section{Analyses}

Differences between participants with and without a diagnosis of dementia were assessed with t-tests; relationships among variables discussed were assessed by correlational analysis. The predictive effectiveness of Fractal D and MMSE on dementia diagnosis was assessed by logistic regression supplemented by a receiver operator characteristic (ROC) analysis to display correct and incorrect dementia classifications. Confidence limit was set at 0.05.

\section{Results \\ Participant characteristics}

Average full scale MMSE scores trended lower but not significantly lower in site 2, with $t=1.936$, $d f=23$, and $p=0.065$. However, mean ADL scores were significantly lower in site 2 (mean $3.8 \mathrm{SD}=1.2$ ) than in site 1 (mean=5.4 SD=1.8) $(\mathrm{t}=2.583, \mathrm{df}=23$, $\mathrm{p}=0.02)$. Neither Fractal D nor the RAWS$\mathrm{CV}$ validation item differed between the two sites (Fractal D $\mathrm{t}=.181$, $\mathrm{df}=23, \mathrm{p}=$ n.s.; RawsCV Chi-Square $=3.074 \mathrm{df}=1 \mathrm{p}=0.08$ ). How ever, participants in site 1 traveled faster on average $(0.46 \mathrm{~m} / \mathrm{s})$ than site 2 participants $(0.33 \mathrm{~m} / \mathrm{s})(\mathrm{t}=2.096, \mathrm{df}=23, \mathrm{p}=0.047)$. The two sites did not differ in mean distance traveled $(\mathrm{t}=.636, \mathrm{df}=23, \mathrm{p}=\mathrm{n} . \mathrm{s}$.). Across participants the number of ADLs was not related to mean path distance traveled within the monitored area $(r=-0.33, n=25 p=0.11)$ (Table 1).

\section{Fractal D}

Consistent with the hypothesis that more aimless movement would be observed in persons with dementia, Fractal D scores 


\section{Detecting d e mentia}

were significantly higher in the dementia group (mean $=1.37 \mathrm{SD}=0.06$ ) than in the no dementia diagnosis group (mean $=1.25$, $\mathrm{SD}=0.07) \quad(\mathrm{t}=4.56, \mathrm{df}=23, \mathrm{p}<0.001) . \quad \mathrm{ADL}$ levels did not differ by dementia status (overall mean=4.7, groups $t=0.48, d f=23 p=0.64$ ). Travel speed was significantly lower in persons with dementia $(0.33 \mathrm{~m} / \mathrm{s})$ than in those with no diagnosis $(0.50 \mathrm{~m} / \mathrm{s}) \quad(\mathrm{t}=3.13, \mathrm{df}=23$, $\mathrm{p}<0.01$ ) (Table 1).

\section{Relationships}

A Chi square test on the RAWS-CV validation item vs. diagnosis of dementia was nonsignificant (Chi square $=0.89 \mathrm{df}=1 \mathrm{p}=0.35$ ) (Table 2). Neither MMSE nor Fractal D reliably differed from normal for persons identified as wanderers although a strong trend in that direction for both measures was observed $(M M S E t=1.88, d f=23 \mathrm{p}=0.07$; Fractal $\mathrm{D} t=1.92, \mathrm{df}=23 \mathrm{p}=0.07)$. Correlation coefficients of Fractal D and the RAWS-CV subscales (persistent walking, repetitive walking, eloping behavior, spatial disorientation and negative outcomes) were non-significant. A correlation coefficient computed between the total MMSE scores and Fractal D was significant ( $r=-0.44, n=25, p=0.03$ ), extending prior observations ${ }^{15}$ to a second research site. The mean MMSE level for participants having a diagnosis of dementia was significantly lower; $13.4(\mathrm{SD}=7.9)$ than for those with no diagnosis; $23.2(\mathrm{SD}=4.7)(\mathrm{t}=3.639$, $d f=23 p=0.001$ ).

\section{Individual MMSE -Fractal D relationships}

Only two MMSE subscales correlated significantly with Fractal D; the Geographic Orientation subscale $(r=-0.66, n=25, p<0.001)$ which was hypothesized to show this relationship, and the Temporal Orientation subscale which was marginally negatively correlated with Fractal D $(r=-0.39, n=25, p=0.05)$.

A logistic regression predicting dementia diagnosis revealed Fractal $D$ was a significant predictor, $(B=0.266, d f=1, p=0.007)$. The estimated odds of dementia increased approximately 30\% with each unit increase in Fractal D scores. The robust HosmerLemeshow goodness-of-fit statistics (appropriate for small sample sizes), showed no evidence of lack of fit for this model (Chi square=8.759, $p=0.188, d f=6) . \quad A$ logistic regression of dementia diagnosis on MMSE was significant $(B=-0.354, d f=1, p=0.035)$. The estimated odds ratio for the MMSE was 0.702 , that is, odds of dementia increased by approximately $30 \%$ when the MMSE full scale score decreased by 1 unit. The robust Hosmer-Lemeshow goodness-of-fit statistics showed no evidence of lack of fit for this model (Chi square $=3.238, p=0.663, d f=5)$.

Cross classification tables were generated based on the model to predict the probability of dementia for each subject using a cutoff of 0.5. Fractal D was associated with a slightly higher overall percentage of correct

Table 2: Pearson correlation matrix of dementia diagnosis, MMSE (Mini Mental State Exam), ADLs requiring help, and movement parameters; 2 -tailed tested for significance; ${ }^{* *}=p<0.01 ;{ }^{*}=0.01<p<0.05$

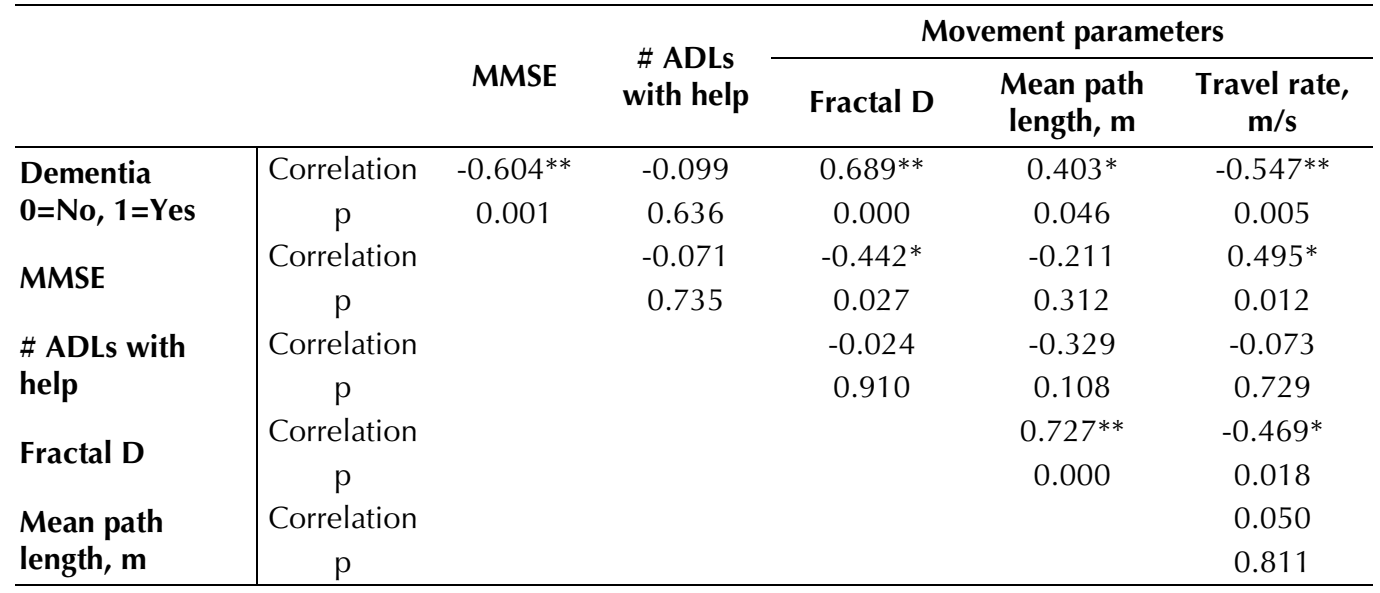




\section{Detecting dementia}

Table 3. Results of step 1 of the logistic regression of MMSE (Mini Mental State Exam) and Fractal D as predictors of the diagnosis of dementia; B=Beta; S.E.=Standard Error.; Wald=Wald Test ${ }^{33}$; $d f=$ degrees of freedom; Sig.=Significance.; C.I.=Confidence interval

\begin{tabular}{|c|c|c|c|c|c|c|c|c|}
\hline & \multicolumn{8}{|c|}{ Variables in the equation } \\
\hline & \multirow{2}{*}{ B } & \multirow{2}{*}{ S.E. } & \multirow{2}{*}{ Wald } & \multirow{2}{*}{ df } & \multirow{2}{*}{ Sig. } & \multirow{2}{*}{$\operatorname{Exp}(B)$} & \multicolumn{2}{|c|}{$95 \%$ C.I. of $\operatorname{Exp}(B)$} \\
\hline & & & & & & & Lower & Upper \\
\hline MMSE & -0.180 & 0.131 & 1.902 & 1 & 0.168 & 0.835 & 0.646 & 1.079 \\
\hline Fractal D & 0.201 & 0.105 & 3.682 & 1 & 0.055 & 1.223 & 0.996 & 1.502 \\
\hline Constant & 122.894 & 14.788 & 2.397 & 1 & 0.122 & 0.000 & & \\
\hline
\end{tabular}

prediction of the dementia groups to which the subjects belonged (84\% versus $80 \%$ ) compared to the MMSE; however, both tests were similar in their sensitivity $(85.7 \%)$.

A single logistic regression including MMSE and Fractal $\mathrm{D}$ as predictors was performed to assess potential confounding. Fractal D is marginally significant as a predictor of dementia $(p=0.055)$, while MMSE is not $(p=0.168)$ (Table 3). Results of the HosmerLemeshow test of the overall model shows no significant lack of fit $(p=0.175)$ and given the small sample size, we evaluated multicollinearity effects; the correlation of estimates for both predictors was 0.294. Therefore multicollinearity probably played a negligible role in the non-significance of the MMSE in the model. The MMSE does not seem to explain any variation in dementia outcome in a significant way over and above that already explained by Fractal $D$, which may explain the lack of a demonstrable difference in the overall percentages of correct classification by Fractal D alone (84\%) versus in combination with the MMSE (84\%).

The diagnostic accuracies of Fractal D and MMSE in their classification diagnosis of dementia (yes or no) were compared using receiver operating characteristic (ROC) curve analysis (Figure 2), where presence or absence of dementia defined according to prior clinical diagnostic assessment is used as the 'reference standard'. The test results of both Fractal D and MMSE being continuous variables may make the choice of optimal cutoff points difficult. ROC curve analysis serves as an appropriate and objective statistical method to assess accuracies of these two diagnostic tests. Because of the relatively small sample size and presence of tied predicted values for dementia classification, a binomial ROC curve was calculated for each test as a 'smooth fit' to the empirical ROC curve (Figure 2), to facilitate the choice of the best cutoff point. As seen from the figure, the approximate best cutoff point overall for Fractal D lies between a sensitivity, or true positive rate, of $80-95 \%$ with corresponding false positive error rates of $20-40 \%$. At the same sensitivity range the approximate false positive error rates for MMSE fall between $25-60 \%$.

\section{Discussion}

There were four major findings in the present study. First, an elevated Fractal D predicts a diagnosis of dementia. The correlations of RAWS-CV with Fractal D and MMSE although in the predicted direction were not statistically reliable. The correlation between number of ADLs requiring assistance and Fractal D were not significant (Table 2),

Second, the total MMSE score was, as anticipated, a significant predictor of dementia and significantly negatively correlated with Fractal D. MMSE scores were not significantly related to the number of ADLs requiring assistance. When both MMSE and Fractal D were entered as predictors of dementia in a logistic regression, the MMSE's contribution was rendered non-significant. A subsequent ROC analysis showed that given a sensitivity of 0.80 for both measures, the false alarm rate for the MMSE was 0.27 as opposed to 0.18 for Fractal D; there were 6 dementia diagnostic misclassifications using the MMSE as opposed to only 2 for Fractal D. Inspection of the MMSE and Fractal D scores shown in Table 1 reveals that the misclassification of demented vs. non-demented persons by the MMSE is greatest for MMSE values ranging from 19-21; above and below those values the MMSE's performance improves. 


\section{Detecting dementia}

ROC Curve: Fractal-D

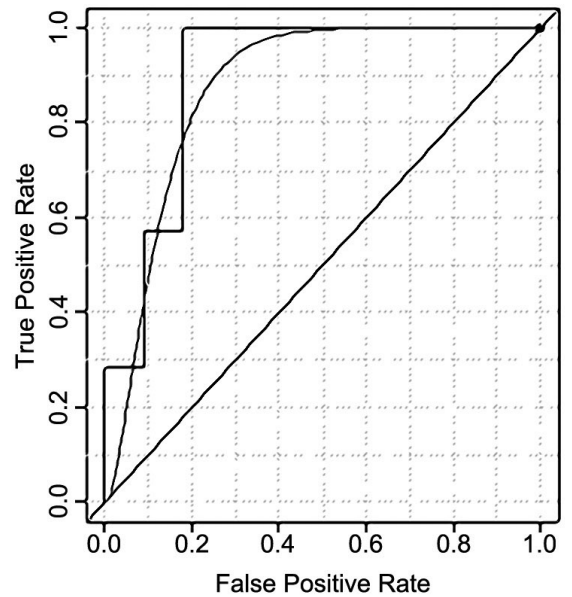

ROC Curve: MMSE

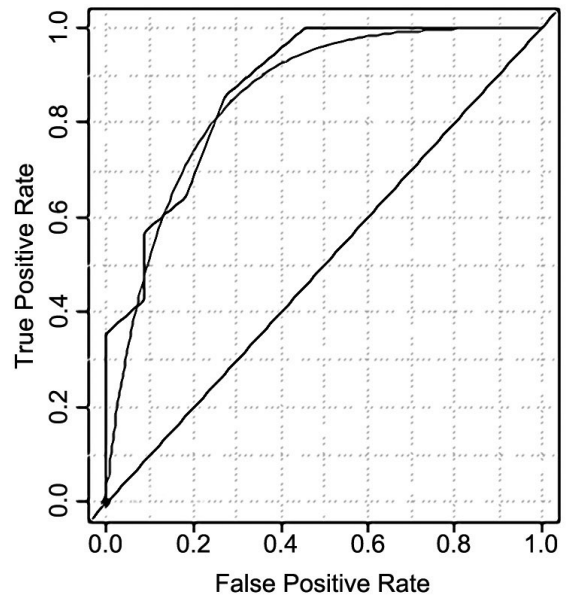

Figure 2. Receiver operator curves for the prediction of dementia diagnosis using Fractal D vs. MMSE (Mini Mental State Exam)

Third, the average rate of travel and mean path length, although significantly correlated with the diagnosis of dementia (Table 2), did not improve upon variance accounted for in the logistic regression which included predictors Fractal D and MMSE. These variables are also significantly correlated with Fractal D (Table 2). Rate of travel is negatively correlated with Fractal $\mathrm{D}$ because higher path tortuosity requires longer times to traverse a given path. Rate of travel provides no information about location. In the absence of changes in path direction, path distance can provide information about location, but because changes in direction of successive paths do occur, the correct designation of location must include changes in path direction: the core element of Fractal D.

Hayes and colleagues ${ }^{7}$ estimated walking speed in elderly persons living alone in their homes over a six month period. The average time required to traverse one meter was the same in a group with mild cognitive decline as in a non-impaired group but the coefficient of variation in the former group was twice that of the latter. Our finding that rate of travel is positively correlated with MMSE scores is consistent with their findings. However, in the present study the mean rate of travel was $0.49 \mathrm{~m} / \mathrm{s}$ and $0.31 \mathrm{~m} / \mathrm{s}$ in the non-demented and demented groups, respectively. The range of travel rates in the present study $(0.15-0.70 \mathrm{~m} / \mathrm{s})$ brackets the $0.56 \mathrm{~m} / \mathrm{s}$ average reported by Hayes et al. ${ }^{7}$. Although the differences could reflect differences in the method of estimating travel rate, the most important factors include the degree of help in ADLs required by participants in the present study and differences in level of cognitive impairment in the two studies. Hayes et al. ${ }^{7}$ defined mild cognitive impairment using the Clinical Dementia Rating Scale; none of their participants had MMSE scores lower than 24.

The fourth finding was that only the Geographical Orientation item of the MMSE was strongly and significantly related to Fractal D while the temporal orientation item was weakly but still significantly associated with Fractal D. The total MMSE score reflects a multidimensional construct of cognitive functioning making it a reliable tool for evaluating general cognitive function. Increased path tortuosity in familiar spaces may reflect a decline in cognitive functions controlling navigation, and the strong association of the MMSE's Geographic Orientation item with Fractal D may be tapping that dimension.

Finally, the anticipated correlation between Fractal D and the RAWS-CV validation item 'is this person a wanderer?' $(r=0.35, n=25$, $p=0.08$ ) was not significant but in the expected direction. Neither were the correla- 


\section{Detecting d e me ntia}

tions between Fractal D and the five RAWSCV subscales significant.

\section{Movement ecology paradigm}

The movement ecology paradigm provides a framework for interpreting the results of this study. It characterizes four major research domains related to movement ${ }^{21}$. The first two, 'biomechanical' and 'cognitive', focus on the basic mechanisms underlying movement, specifically motion and navigational capacities defined earlier. The third, 'random' domain focuses on the movement patterns, and the fourth, 'optimality' domain focuses on interactions between the internal state (why move) and the external environment. The present study bridges the cognitive and random domains by examining movements generated by persons who retain scant memory of their local environment. It is an example, in Nathan's terms, of "...new approaches to explore potential links between intermittent locomotion, reorientation behavior and search efficiency... particularly valuable for identifying different movement phases and distinct behaviors from movement paths" ${ }^{21 p 19058}$.

In their analysis of the role of executive function and attention on human gait disorders, Yogev-Seligmann and colleagues ${ }^{32}$ identify five interrelated cognitive functions spanning four movement domains: (i) volition: setting goals for movement; (ii) self-awareness: placing oneself in the environment to be traversed; (iii) planning: organizing steps needed to carry out the activity; (iv) response inhibition: ignoring distractions to intended movement; and (v) response monitoring: comparing ongoing and intended actions. Their framework provides a means for understanding how the extra cognitive burden imposed by dual tasks affects gait and provides opportunities for future investigations to discover links between path tortuosity and independent neuropsychological measures of cognitive functions involved in gait disorders.

To our knowledge this is the first report linking dementia diagnosis to the tortuosity of movement paths recorded in residential set- tings. The paths were generated by traversing between bedrooms and dining areas or when engaged in going to social and recreational activities such as watching television or hobbies in the monitored space. We employed a transponder technology to track locomotion, and quantify its aimless component mathematically as movement tortuosity (Fractal D). Path tortuosity quantified as Fractal D may represent dementia sufferers' difficulty remembering spatial cues essential for navigating familiar environments irrespective of means of locomotion. Among the many undesirable consequences of dementia are increasingly irregular gait, heightened fall risk, and becoming lost in familiar places.

\section{Study limitations}

The successful use of this telesurveillance system in two settings attests to its reliability and accuracy. In this application, a mixed media TCP/IP connection from the investigator's computer allows remote detection and management of software or hardware issues on the onsite computers, which process, store and forward the collected data. In almost all cases of data transmission failures, it was the result of a participant not wearing the transponder, and was remedied by notifying staff at the ALF who located and replaced the tag. The long term goal of this project: automatic detection of changes in Fractal D and other measures of locomotion related to health risks with subsequent reporting these results to the ALF administrator and/or clinician, so action may be taken in a timely manner, has come a step nearer.

We placed the tags on the participants' wrists. Would Fractal D measure movements of the wrist relative to the body, rather than of the body itself? The range of scales used in the Fractal D estimates minimized this possibility. The range of spatial scales used was determined by the mean step size and the scaling constants - this resulted in an average range of scales of $0.25-5 \mathrm{~m}$. Most of that range is larger than the size of wrist movements relative to the body, and thus Fractal D would mostly respond to movement of the whole body itself. 


\section{Detecting dementia}

One might also wonder how variability in activity might affect our estimates; it depends on the type of variability. One type is variability in time spent moving - i.e. moving vs. not moving. This would not affect Fractal $D$ estimates because the technique is based on distances and directions, not on the time taken to go from place to place. Thus, only the movement path itself is relevant, not whether the participant stopped and started while travelling along the path. Another type of variability is variability in path tortuosity i.e., straight sections then tortuous sections. This would also not affect Fractal D estimates because they are based on mean tortuosity. A final type of variability is the predictability of path direction; this is specifically what Fractal D measures. A low Fractal D value means the path remains quite straight, while a high $\mathrm{D}$ value means path direction varies and is unpredictable. Our results suggest that dementia decreases predictability of movement direction.

\section{Acknowledgments}

Support was provided by Johnnie B. Byrd, Sr. Alzheimer's Research Center \& Research Institute under ARG-2007-34 and AHRQ R21 HS18205-01, 'Evaluation and Integration of an Automatic Fall Prediction System'. The authors acknowledge the assistance of Dr. Drew Petkus, Ms. Lindsay Iser, Dr. Victor Molinari, and Scott Barnett as well as the anonymous reviewers of an earlier version of the manuscript.

\section{References}

1. Camicioli R, Howieson D, Oken B, Sexton $\mathrm{G}$, Kaye J. Motor slowing precedes cognitive impairment in the oldest old. Neurology 1998;50(5):1496-1498

2. Ramakers IHGB, Visser PJ, Aalten P, Boesten JHM, Metsemakers JFM, Jolles J, Verhey FRJ. Symptoms of Preclinical Dementia in General Practice up to Five Years Before Dementia Diagnosis. Dementia \& Geriatric Cognitive Disorders 2007;24(4):300-306; doi:10.1159/000107594

3. Johnson DK, Storandt M, Morris JC, Galvin JE. Longitudinal study of the transition from healthy aging to Alzheimer disease. Archives of Neurology 2009;66(10):1254-1259

4. Sanders A, Holtzer R, Lipton R, Hall C, Verghese J. Egocentric and Exocentric Navigation Skills in Older Adults. Journals of Gerontology Series A: Biological and Medical Sciences 2008;63(12):1356; doi:10.1001/
Limitations of this study include a lack of detailed information about the severity, type, and duration of the diagnosis of dementia. It is possible that other unmeasured functional limitations or diseases related to movement contributed to the observed differences in Fractal D in demented and non-demented groups. In view of the finding that only the MMSE geographical orientation subscale was strongly and significantly related to Fractal D, it will be of interest to obtain other measures of visuospatial functioning in future studies.

\section{Future research}

Future research should also focus on detecting other components of wandering than aimless motion quantified by Fractal D, such as lapping or repetitive walking back and forth (pacing) in order to compare their spatial variability with temporal variability observed in formal assessments of gait and balance using prescribed courses of movements.

\section{archneurol.2009.158}

5. Algase D, Struble L. Wandering behavior: What, why, and how. In: Buckwalter K, editor, Geriatric Mental Health Nursing: Current and Future Challenges. Thorofare: Slack; 1992; pp 61-74

6. Algase DL, Son GR, Beattie E, Song JA, Leitsch S, Yao L. The interrelatedness of wandering and wayfinding in a community sample of persons with dementia. Dementia \& Geriatric Cognitive Disorders 2004;17(3):231-239; doi:10.1159/000076361

7. Hayes TL, Pavel M, Kaye JA. An unobtrusive in-home monitoring system for detection of key motor changes preceding cognitive decline. The 26th Annual International Conference of the IEEE Engineering in Medicine and Biology Society 2004;4:24802483; doi:10.1109/IEMBS.2004.1403715

8. Pavel M, Hayes TL, Adami A, Jimison H, Kaye J. Unobtrusive assessment of mobility. 2006 International Conference of the IEEE Engineering in Medicine and Biology Society;2006;1:6277-6280; doi:10.1109/ IEMBS.2006.260301

9. Bernstein N. The coordination and regulation of movements. London: Pergamon, 1967

10. Hausdorff JM. Gait dynamics, fractals and falls: Finding meaning in the stride-to-stride fluctuations of human walking. Human Movement Science 2007;26(4):555-589; 


\section{Detecting dementia}

doi:10.1016/j.humov.2007.05.003

11. Verghese J, Holtzer R, Lipton RB, Wang C. Quantitative Gait Markers and Incident Fall Risk in Older Adults. Journals of Gerontology Series A: Biological and Medical Sciences 2009;64A(8):896-901

12. Verghese J, Lipton R, Hall C, Kuslansky G, Katz M, Buschke H. Abnormality of Gait as a Predictor of Non-Alzheimer's Dementia. New England Journal of Medicine 2002;347(22):1761-1768; doi:10.1056/NEJMoa020441

13. Kearns W, Nams V, Fozard J. Wireless fractal estimation of tortuosity in movement paths related to cognitive impairment in assisted living facility residents. Methods in Information Medicine 2010;49(6):592-598; doi:10.3414/ME09-01-0079

14. Kearns WD, Algase D, Moore DH, Ahmed S. Ultra wideband radio: A novel method for measuring wandering in persons with dementia. Gerontechnology 2008;7(1):4857; doi:10.4017/gt.2008.07.01.005.00

15. Kearns W, Fozard JL. Evaluation of Wandering by Residents in an Assisted Living Facility (ALF) using Ultra Wideband Radio RTLS. The Journal of Nutrition, Health \& Aging 2009;13:S54

16. Nams VO, Bourgeois M. Fractal analysis measures habitat use at different spatial scales: an example with American marten. Canadian Journal of Zoology 2004;82(11):1738-1747; doi:10.1139/z04-167

17. Merory JR, Wittwer JE, Rowe CC, Webster KE. Quantitative Gait Analysis in Patients With Dementia With Lewy Bodies and Alzheimer's Disease. Gait \& Posture 2007;26(3):414-419; doi:10.1016/j. gaitpost.2006.10.006

18. Algase DL, Moore $\mathrm{DH}$, Vandeweerd C, Gavln-Dreschnack DJ. Mapping the maze of terms and definitions in dementia-related wandering. Aging \& Mental Health 2007; 11(6):686-698; doi:10.1080/13607860701366434

19. Nelson AL, Algase DL. Appendix B. Measurement Tools for Wandering. Revised Algase Wandering Scale-Community Version. In: Nelson AL, Algase DL, editors, Evidence-based protocols for managing wandering behavior. New York: Springer; 2007; pp 385-397

20. Luis CA, Brown LM. Neuropsychological correlates of wanderers. In: Nelson $\mathrm{AL}, \mathrm{Al}$ gase DL, editors, Evidence-based protocols for managing wandering behavior. New York: Springer; 2007; pp 65-74

21. Nathan R, Getz WM, Revilla E, Holyoak M, Kadmon R, Saltze D, Smous PE. A movement ecology paradigm for unifying organismal movement research. Proceedings of the National Academy of Sciences 2008;105(49):19052-19059; doi:10.1073/ pnas.0800375105

22. Snyder L, Rupprecht P, Pyrek J, Brekhus $\mathrm{S}$, Moss T. Wandering. Gerontologist 1978;18(3):272

23. De Leon MJ, Potegal M, Gurland B. Wandering and parietal signs in senile dementia of Alzheimer's type. Neuropsychobiology 1984;11(3):155-157; doi:10.1159/000118069

24. Ryan JP, McGowan J, McCaffrey N, Ryan GT, Zandi T, Brannigan GG. Graphomotor perseveration and wandering in Alzheimer's disease. Journal of Geriatrics, Psychiatry and Neurology 1995;8(4):209-212

25. Passini R, Rainville C, Marchand N, Joanette Y. Wayfinding in dementia of the Alzheimer type: planning abilities. Journal of Clinical and Experimental Neuropsychology 1995;17(6):820-832; doi:10.1080/01688639508402431

26. Jensen J, Nyberg L, Gustafson Y, LundinOlsson L. Fall and Injury Prevention in Residential Care-Effects in Residents with Higher and Lower Levels of Cognition. Journal of the American Geriatrics Society 2003;51(5):627-635; doi:10.1034/j.16000579.2003.00206.x

27. Rubenstein LZ. Falls in older people: epidemiology, risk factors and strategies for prevention. Age and Ageing 2006;35(Suppl 2):ii37-ii41; doi:10.1093/ageing/afl084

28. Ubisense, Inc. Series 7000 Compact Tag; www.ubisense.net/en/resources/factsheets/ series-7000-compact-tag.html; retrieved: May 19, 2011

29. Fractal computer program Nams V, Version v3.16. Nova Scotia Agricultural College; 1997-2001

30. Nams VO. Improving accuracy and precision in estimating fractal dimension of animal movement paths. Acta Biotheoretica 2006;54(1):1-11; doi:10.1007/s10441-0065954-8

31. Craighead J. Using Fractal Dimension to Assess Robot Operator Skill. 2009 IEEE International Workshop on Safety, Security \& Rescue Robotics (SSRR 2009); 2009; doi:10.1109/SSRR.2009.5424146

32. Yogev-Seligmann G, Hausdorff JM, Giladi $\mathrm{N}$. The Role of Executive Function and Attention in Gait. Movement Disorders 2008;23(3):329-342; doi:10.1002/mds.21720

33. Buse E. The likelihood ratio, Wald, and language multiplier tests: An expository note. American Statistician 1982;36(3):153-157 